\title{
BMJ Open Sleep: population epidemiology and concordance in Australian children aged 11-12 years and their parents
}

\author{
Lisa Matricciani, ${ }^{1,2}$ Francois Fraysse, ${ }^{1}$ Anneke C Grobler, ${ }^{\circledR 2,3}$ Josh Muller, $^{2}$ \\ Melissa Wake, ${ }^{\oplus, 3,4}$ Timothy Olds $^{\oplus 1,2}$
}

To cite: Matricciani L, Fraysse F, Grobler AC, et al. Sleep: population epidemiology and concordance in Australian children aged $11-12$ years and their parents. BMJ Open 2019;9:127-135. doi:10.1136/ bmjopen-2017-020895

- Prepublication history for this paper is available online. To view these files, please visit the journal online (http://dx.doi. org/10.1136/bmjopen-2017020895).

Received 29 November 2017 Revised 2 August 2018 Accepted 4 April 2019

Check for updates

(c) Author(s) (or their employer(s)) 2019. Re-use permitted under CC BY-NC. No commercial re-use. See rights and permissions. Published by BMJ.

${ }^{1}$ Sansom Institute, Alliance for Research in Exercise, Nutrition and Activity (ARENA), University of South Australia, Adelaide, South Australia, Australia ${ }^{2}$ Murdoch Children's Research Institute, Parkville, Victoria, Australia

${ }^{3}$ Department of Paediatrics, The University of Melbourne, Parkville, Victoria, Australia ${ }^{4}$ Department of Paediatrics and The Liggins Institute, The University of Auckland, Auckland, New Zealand

Correspondence to Professor Melissa Wake; melissa.wake@mcri.edu.au

\section{ABSTRACT}

Objectives To describe objectively measured sleep characteristics in children aged 11-12 years and in parents and to examine intergenerational concordance of sleep characteristics.

Design Population-based cross-sectional study (the Child Health CheckPoint), nested within the Longitudinal Study of Australian Children.

Setting Data were collected between February 2015 and March 2016 across assessment centres in Australian major cities and selected regional towns.

Participants Of the participating CheckPoint families $(n=1874)$, sleep data were available for 1261 children (mean age 12 years, 50\% girls), 1358 parents (mean age 43.8 years; $88 \%$ mothers) and 1077 biological parentchild pairs. Survey weights were applied and statistical methods accounted for the complex sample design, stratification and clustering within postcodes.

Outcome measures Parents and children were asked to wear a GENEActive wrist-worn accelerometer for 8 days to collect objective sleep data. Primary outcomes were average sleep duration, onset, offset, day-to-day variability and efficiency. All sleep characteristics were weighted $5: 2$ to account for weekdays versus weekends. Biological parentchild concordance was quantified using Pearson's correlation coefficients in unadjusted models and regression coefficients in adjusted models.

Results The mean sleep duration of parents and children was $501 \mathrm{~min}$ (SD 56) and $565 \mathrm{~min}$ (SD 44), respectively; the mean sleep onset was 22:42 and 22:02, the mean sleep offset was $07: 07$ and $07: 27$, efficiency was $85.4 \%$ and $84.1 \%$, and day-to-day variability was $9.9 \%$ and $7.4 \%$, respectively. Parent-child correlation for sleep duration was $0.22(95 \% \mathrm{Cl}$ 0.10 to 0.28 ), sleep onset was 0.42 ( 0.19 to 0.46 ), sleep offset was 0.58 ( 0.49 to 0.64$)$, day-to-day variability was 0.25 ( 0.09 to 0.34 ) and sleep efficiency was 0.23 ( 0.10 to 0.27 ).

Conclusions These normative values for objective sleep characteristics suggest that, while most parents and children show adequate sleep duration, poor-quality (low efficiency) sleep is common. Parent-child concordance was strongest for sleep onset/offset, most likely reflecting shared environments, and modest for duration, variability and efficiency.

\section{INTRODUCTION}

Sleep is essential for health and well-being. ${ }^{1}$ The importance of sleep duration, as well as sleep timing, variability and quality (often

\section{Strengths and limitations of this study}

This study used valid, reliable, objective, free-living measures of child and parent sleep.

- We report, for the first time, concordance in sleep time characteristics other than duration and timing (onset and offset), including sleep efficiency and day-to-day variability.

- The sample represents a limited age range for children (11.0-12.9 years) and adults (midlife).

- Intergenerational concordance of parent and child sleep was examined for only one of the child's parents, predominantly mothers.

measured objectively as sleep efficiency), is increasingly being recognised. ${ }^{2-4}$ Characteristics of sleep time have been associated with a wide range of health outcomes, including cognitive function, ${ }^{56}$ mood, ${ }^{7}$ weight status, ${ }^{8}$ diabetes $^{9}$ and cardiovascular risk..$^{10} 11$

Despite the importance of sleep, concerns have been raised that many adults and children report inadequate, irregular and poor-quality sleep. ${ }^{12-15}$ The National Sleep Foundation (NSF) ${ }^{16} 17$ found that $45 \%$ of Americans reported poor or insufficient sleep that affects their daily activities at least once a week, while $58 \%$ of 15-17year olds reported sleeping less than 7 hours per night, and $17 \%$ rated their sleep quality as fair or poor. Similarly, the 2016 Sleep Healthy Survey of Australian Adults ${ }^{18}$ found that $33 \%-45 \%$ of Australian adults said that they were affected by poor sleep (inadequate duration or quality). In a global review of 41 studies examining children's sleep, Gradisar and colleagues $^{19}$ found $53 \%$ of samples reported insufficient sleep (by their definition of $<8$ hours) on school nights.

Although relatively less examined and understood, sleep variability, the degree to which an individual's night time sleep duration differs across a period of time, is 
increasingly being recognised for both physical and mental health. ${ }^{20}$

Given these concerns, interest has risen in better understanding determinants of sleep characteristics. Patterns of sleep are likely to be partly environmental ${ }^{21}$ and partly genetic. ${ }^{22}{ }^{23}$ Environmental factors may be considered in terms of shared and non-shared parent-child environments. Shared local environmental factors, such as traffic noise, can affect sleep characteristics, such as sleep efficiency. ${ }^{24}$ Similarly, social factors, ${ }^{25}$ such as parenting style, household chaos and family routines, may also play a role, as may child-to-parent effects (ie, reverse causation), particularly when the child is young. Non-shared environmental factors, such as parental work schedules ${ }^{26}$ and school commitment, ${ }^{27}$ are also known to affect sleep characteristics. Genes are also likely to account for some of the interindividual variability in sleep duration. ${ }^{28}$ Personality factors with a strong genetic basis, such as higher levels of conscientiousness, openness and neuroticism, have been associated with shorter sleep. ${ }^{29}$ The rate and timing of melatonin and serotonin release, which are important regulatory hormones of the sleep/wake cycle, are also partially under genetic control. Shared environments and genetics should ensure a degree of concordance between parental and child sleep habits.

Understanding the concordance between parental and child sleep habits can cast some light on the relative importance of environmental and genetic contributors of sleep characteristics. For example, if the concordance is very weak, then shared environmental and genetic components must be relatively small. Similarly, if the concordance is not attenuated when covariates relating to shared or non-shared environmental factors are included, then it is likely that genetics may have a stronger contribution.

To date, few studies have examined the concordance between parents' and children's sleep, most of which examine self-reported or proxy-reported sleep measures. Gau and Merikangas, ${ }^{30}$ in a study of 1479 children aged 10-16 years, found weak correlations between self-reported parent and children's bedtimes (weekdays, $r=0.08$; weekends, $r=0.22$ ), wake times (weekdays, $r=0.02$; weekends, $r=0.26)$ and sleep duration $(r=0.02)$, with notable day-type differences. In a larger study of 4470 parents and their children aged 6-12years, Zhang and colleagues ${ }^{31}$ also reported weak self-reported sleep-timing correlations; however, stronger correlations were observed for mother-child (bedtime, $r=0.27$; wake time, $r=0.28$ ) pairs, compared with father-child (bedtime, $\mathrm{r}=0.10$; wake time, $\mathrm{r}=0.08$ ) pairs. Iwata and colleagues ${ }^{32}$ examined 47 parent-child pairs and found that self-reported parent rise times were associated with their 5-year-old child's objectively measured rise times (weekdays, $r=0.38$; weekends, $r=0.39$ ) but not sleep bedtimes or sleep period times.

Although self-reported sleep duration does correlate with objectively measured sleep, the level of agreement depends on the phrasing of the question and is limited by recall and social desirability bias. ${ }^{33}$ Other important elements of sleep, including estimates of the number and duration of nocturnal awakenings, correlate poorly with objective measures. ${ }^{33}$ Concerns about the societal as well as individual impacts of poor, inadequate and inconsistent sleep on populations as well as individuals justify accurate measurement of multiple sleep characteristics in population-based studies.

Ohayon and colleagues ${ }^{34}$ recently conducted a systematic literature review of 65 studies covering 3577 participants aged 5-102 years in an attempt to identify age-related changes in objectively recorded sleep across a lifespan. This review found that objectively measured sleep duration varied between 360 and $460 \mathrm{~min}$ for adults aged 40-50 years and between 450 and $550 \mathrm{~min}$ for children aged 11-12 years. Sleep efficiency, often defined as the percent of time spent asleep between sleep onset and sleep offset, also varied across studies, ranging from $87 \%$ to $96 \%$ for adults aged $40-50$ years and from $89 \%$ to $98 \%$ for children aged 11-12 years, within the recommended range of greater than $85 \% .{ }^{34}{ }^{35}$ Though comprehensive, Ohayon and colleagues' ${ }^{34}$ review acknowledged a scarcity of objectively measured sleep studies among school-aged children, adolescents and middle-aged parents. Further studies that present normative, objectively measured sleep are needed for these age groups.

The current study examines objectively measured time-use data collected as part of the cross-sectional, observational CheckPoint study. Among this sample of Australian children and their parents, drawn from a national longitudinal study that was initially population representative, we aimed to

1. Assess the distribution of sleep-time characteristics (sleep duration, onset, offset, day-to-day variability and efficiency) in

a. Australian children aged 11-12 years, and

b. Their parents.

2. Describe the extent of intergenerational concordance for sleep-time characteristics.

\section{METHODS}

\section{Study design and participants}

Data examined in this study were collected between February 2015 to March 2016 as part of the Child Health CheckPoint study, a one-off, comprehensive physical health and biomarker cross-sectional study nested between waves 6 and 7 of the Longitudinal Study of Australian Children (LSAC) at child age 11-12 years. The LSAC study commenced in 2004, when two cohorts (B and $\mathrm{K}$-the latter not relevant to this paper) were recruited who have since been followed up biennially. ${ }^{36}$ Details of the initial LSAC study design and recruitment are outlined elsewhere. ${ }^{37} 38$

Of the 8921 families contacted to be part of the LSAC B cohort, 5107 families (57\%) agreed to take part in the first wave of data collection in 2004; 3764 families participated in wave 6 in 2014. During the wave 6 LSAC home visit, B cohort families were introduced to the upcoming 
Child Health CheckPoint and were asked to consent to their contact details being shared with the CheckPoint team. A total of 3513 families provided permission to receive an information pack via mail, and an information and recruitment phone call regarding the CheckPoint study ( $78 \%$ of the wave 6 cohort and $69 \%$ of the original cohort). Of the families agreeing to receive information about the CheckPoint study, 1874 families took part (53\% of eligible participants, $42 \%$ of the wave 6 cohort and $37 \%$ of the original cohort). Details regarding the CheckPoint study design and methods are available elsewhere. ${ }^{39} 40$

\section{Consent}

The attending parent/caregiver provided written informed consent for themselves and their child to participate in the study.

\section{Procedure}

The specialised CheckPoint assessment centre sequentially visited seven Australian major cities and eight smaller regional centres between February 2015 and March $2016 .{ }^{39}$ Each participating child attended the centre with one parent or caregiver (usually the biological mother) at which both participated in a wide range of measures relevant to non-communicable disease. Those families $(n=378)$ who could not arrange a visit were offered a home visit. Sleep data were collected via accelerometers fitted at the end of the visit at the 'End Games' station by a trained research assistant. At peak centre throughput (mainly school holidays), accelerometer demand sometimes exceeded supply. Data for $67(4.8 \%)$ children and $70(4.9 \%)$ parents were therefore obtained from accelerometers that were mailed after the visit. Participants were asked to remove their accelerometers after eight continuous days of wear and to return them with their completed activity monitor cards (see below) using the prepaid postal bag provided.

\section{Measures}

Objectively measured sleep characteristics were collected using triaxial, wrist-worn GENEActiv accelerometers. Using a standardised protocol, the research assistant spoke with each parent and child at the same time, fitted the accelerometer to their non-dominant wrist, explained the importance of continuous 24-hour wear and asked them to wear it for eight consecutive days. They also showed participants how to complete a self-report record (activity monitor card) marking their bedtimes and wake times, as well as any times that they removed the accelerometer and the reasons for non-wear. Activity monitor card data were transcribed electronically to Research Electronic Data Capture (REDCap) ${ }^{41}$

Acceleration data were recorded at $50 \mathrm{~Hz}$. To remove invalid data, days with $\geq 1000$ min of sedentary time were excluded, because these were deemed to reflect undetected non-wear. We also excluded participants with less than four nights of sleep ${ }^{42}$ recorded and participants with $\leq 200$ min average sleep time.
Sleep-time characteristics were derived from raw accelerometer data, using self-reported records of bedtime and wake time as a guide to locating sleep onset and sleep offset. Data were processed using Cobra custom software developed by coauthor Francois Fraysse. Further details of raw accelerometry data processing have been reported elsewhere. ${ }^{43}$ The sleep algorithm of van Hees and colleagues $^{44}$ was used to detect sleep and wake between self-reported bedtime and get-up time. This method is based on the variability of the orientation of the device and classifies each $5 \mathrm{~s}$ epoch as either sleep or wake. Following this, sleep/wake data were further collapsed into $1 \mathrm{~min}$ epochs to align with the rest of the accelerometry data. Each minute was classified as sleep or wake if it contained a majority of sleep or wake $5 \mathrm{~s}$ epochs, respectively. Minutes containing equal numbers of sleep and wake $5 \mathrm{~s}$ epochs were classified as sleep. Sleep data for the first night were excluded, as recordings started at 23:00.

Following this, the variables below were calculated:

- Sleep onset: the start of the first three consecutive minutes scored as sleep.

- Sleep offset: the end of the last five consecutive minutes scored as sleep.

- Sleep duration: the difference between sleep onset and sleep offset.

- Day-to-day sleep variability: the coefficient of variation of duration.

- Sleep efficiency: the percent of minutes scored as sleep between onset and offset.

Socioeconomic status was derived from the postcode of the participant's primary address using the Socio-Economic Indicators for Areas 2011 Index of Relative Social Disadvantage, which measures relative disadvantage from variables like low income, low educational attainment and unemployment. ${ }^{45}$ The population mean score for Australia is 1000 (SD 100), with higher scores representing less disadvantage. ${ }^{45}$

\section{Statistical analysis}

Population summary statistics were estimated by applying survey weights and procedures, taking clustering in the sampling frame into account. SEs were calculated, taking into account the complex design and weights. More detail on the calculation of weights is provided elsewhere. ${ }^{3946} \mathrm{All}$ sleep characteristics were computed for each individual day, then averaged over days for each valid participant using a 5:2 weighting for weekdays (Sunday-Thursday) and weekend (Friday-Saturday). The key sleep variables of duration and efficiency were similar on weekdays and weekends (see table 1). Therefore, we examined a single mean score for each sleep variable examined, noting, however, that both sleep onset and sleep offset were somewhat later for children and parents at weekends.

Concordance between parents and children was assessed by (1) Pearson's correlation coefficients with 95\% CIs and (2) linear regression with the child variable as the dependent variable and the parent variable as the independent 
Table 1 Sleep variables for weekdays and weekends; all values are mean (SD)

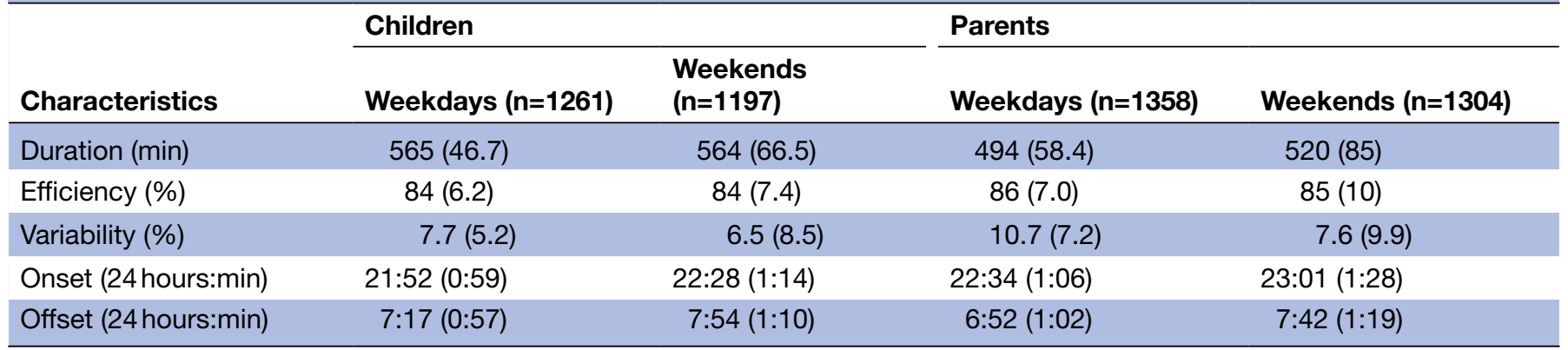

variable, adjusted for parent and child age and sex (in models including both sexes), relative socioeconomic disadvantage and day type (ie, mixed days, weekdays only or weekend/holidays only). In most children $(n=1241)$ and parents $(n=1346)$, measurements were taken on both weekends and weekdays ('mixed' days), while few parents $(n=12)$ and children $(n=20)$ had data on weekdays only, and no parents and children had data on weekends/holidays only. Non-biological caregivers were excluded from these analyses $(n=12)$.

\section{Patient and public involvement}

Because LSAC is a population-based longitudinal study, no patient groups were involved in its design or conduct. To our knowledge, the public was not involved in the study design, recruitment or conduct of LSAC study or its CheckPoint module. Parents received a summary health report for their child and themselves at or soon after the assessment visit. They consented to take part, knowing that they would not otherwise receive individual results about themselves or their child.

\section{RESULTS}

Of the 1874 families who participated in the CheckPoint, sleep data were available for 1261 children, 1358 adults and 1077 biological parent-child pairs (figure 1).

Table 2 outlines the sample characteristics. While the numbers of boys and girls were approximately equal, most parents $(88 \%)$ were mothers. Participants were slightly less disadvantaged than the general Australian population, with the mean Disadvantage Index score around $0.1 \mathrm{SD}$ above the national average and showing a narrower spread (SD 64 vs the national value of 100) such that there were few very disadvantaged participants.

\section{Sleep characteristics}

Table 3 presents the summary statistics for sleep characteristics (sleep duration, onset, offset, day-to-day variability and efficiency) in Australian middle-aged parents and children aged 11-12 years, while figure 2 shows the distributions graphically for sleep duration and efficiency, with lines dropped to represent US-based NSF recommendations. $^{35} 47$

Overall, $6.4 \%$ of the parent sample, but $27.8 \%$ of the child sample, fell below the NSF's recommended range of appropriate sleep duration of 420-540 min and 540-660 min, respectively. The mean sleep efficiency was $85 \%$ for parents and $84 \%$ for children. This equates to $42.3 \%$ of parents and $54.5 \%$ of children falling below the lower end of NSF's ${ }^{35}$ recommended range of $\geq 85 \%$.

Overall, children and parents had a day-to-day sleep variability of $7.4 \%$ and $9.9 \%$, respectively. This equates to an SD in night-time sleep duration of approximately 42-50 min for children and parents, respectively.
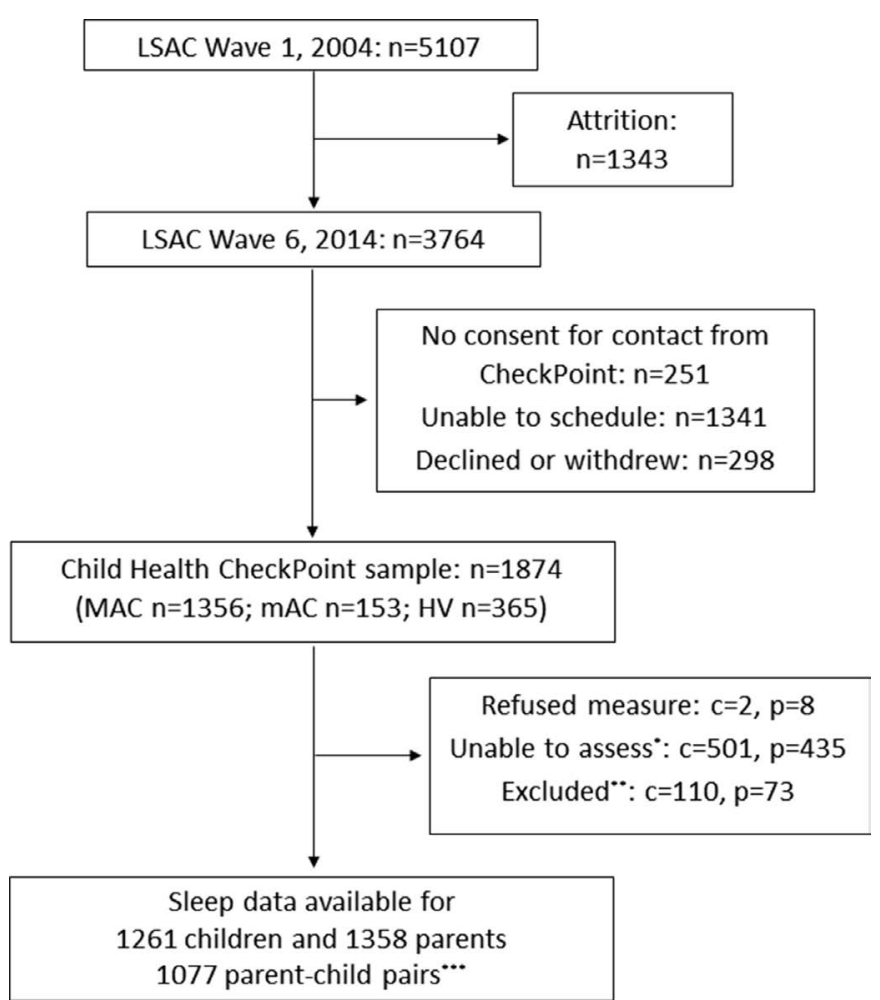

Figure 1 Participant flow chart. *Inability to assess due to equipment failure, poor-quality data or time constraints. ${ }^{\star *}$ Participants excluded if valid days available did not meet the minimum criteria of at least 4 days of any type, $\geq 200$ min sleep and $\leq 1000$ min sedentary time. ${ }^{* \star *}$ Data from 12 nonbiological child-parent pairs excluded from concordance analyses. c, number of children; HV, home visit assessment; LSAC, Longitudinal Study of Australian Children; MAC, main assessment centre; mAC, mini assessment centre; $\mathrm{n}$, number of families; $\mathrm{p}$, number of attending adults. 
Table 2 Participant characteristics (weighted means and SD)

\begin{tabular}{|c|c|c|c|c|c|c|c|c|c|}
\hline \multirow[b]{2}{*}{ Child characteristics } & \multicolumn{3}{|c|}{ Daughters } & \multicolumn{3}{|c|}{ Sons } & \multicolumn{3}{|c|}{ All children } \\
\hline & $\mathbf{n}$ & Mean* & SD* $^{*}$ & $\mathbf{n}$ & Mean* & SD* & $\mathbf{n}$ & Mean* & SD* \\
\hline Age (years) & 629 & 12.0 & 0.4 & 632 & 12.0 & 0.4 & 1261 & 12.0 & 0.4 \\
\hline \multirow[t]{2}{*}{ Disadvantage Index } & 628 & 1010 & 65 & 629 & 1010 & 63 & 1257 & 1010 & 64 \\
\hline & \multicolumn{3}{|c|}{ Mothers } & \multicolumn{3}{|c|}{ Fathers } & \multicolumn{3}{|c|}{ All Parents } \\
\hline Age (years) & 1191 & 43.6 & 5.3 & 167 & 46.3 & 7.1 & 1358 & 43.9 & 5.6 \\
\hline $\mathrm{BMI}\left(\mathrm{kg} / \mathrm{m}^{2}\right)$ & 1183 & 27.9 & 6.5 & 167 & 28.8 & 5.1 & 1350 & 28.0 & 6.4 \\
\hline Disadvantage Index & 1191 & 1012 & 63 & 165 & 1011 & 63 & 1356 & 1012 & 63 \\
\hline
\end{tabular}

Disadvantage Index indicates the Index of Relative Socioeconomic Disadvantage .

*Weighted mean and SD.

BMI, body mass index.

\section{Intergenerational concordance for sleep-time characteristics} Table 4 presents the intergenerational concordance for all sleep-time characteristics. None of the associations were attenuated in adjusted linear regression models, suggesting that the concordance between parents and children did not change when adjusted for age, Disadvantage Index, type of day or sex. Estimates across the characteristics were more consistent for mother-child pairs than father-child pairs, perhaps reflecting the smaller father sample size $(n=128,11.9 \%$ of parent participants $)$ and accordingly larger CIs and greater uncertainty (table 4); nonetheless, mother-child and father-child correlations were strikingly similar for three of the five characteristics (duration, efficiency and sleep offset). Higher correlations both for mother-child pairs and father-child pairs for sleep offset suggest a high degree of household synchronicity, which was also present but less marked for sleep onset.

\section{DISCUSSION}

\section{Principal findings}

To our knowledge, this is the first study to provide normative values and report parent-child concordance of multiple objectively measured sleep-time characteristics (sleep duration, onset, offset, day-to-day variability and efficiency) in a population-based sample.

By current accepted guidelines, sleep duration was adequate for almost all parents but for only $72 \%$ of children aged 11-12years, while close to half of all parents and children experienced poor sleep quality on current recommendations. ${ }^{35}{ }^{47}$ Sleep variability was $7.4 \%$ for children and $9.9 \%$ for parents, equating to an SD in nighttime sleep duration of approximately $42-50 \mathrm{~min}$. We provide estimates of both mother-child and father-child concordance at the population level and show parentchild correlations in all metrics, strongest for sleep offset and sleep onset and for mother-child pairs.

\section{Strengths and weaknesses}

This study reports valid, reliable, objective, free-living measures of child and parent sleep. Data were collected across the same week using the same protocols and devices for children and parents, strengthening our conclusions about parent-child concordance.

Despite study strengths, there are also study limitations. Limitations include the narrow age range for children (11-12 years), precluding generalisation to other childhood ages, the small number of fathers and participation of dyads rather than triads. Further, high attrition rates (53\% of eligible participants took part) may have biased sleep estimates to reflect a relatively advantaged sample.

There are also a number of methodological issues that also need to be considered. First, sleep estimates are known to vary across actigraphy devices and analytical algorithms. For example, hip-worn monitors have been shown to overestimate sleep efficiency and duration when compared with wrist-worn monitors, ${ }^{48}$ and the need for further validity studies on the different algorithms that are integrated in the manufacturers' software has been acknowledged. ${ }^{49}$ Second, it is often difficult to distinguish sleep from non-wear, and so there may be some residual upward bias for sleep duration and efficiency results. The algorithm used to detect sleep in this study has been reported to overestimate sleep by $31 \mathrm{~min}$, when compared with polysomnography, the gold-standard method used to measure sleep. ${ }^{44}$ Lastly, since much of our understanding of sleep stems from self-report data, caution is needed when comparing objective sleep measures to previous literature. Sleep recommendations, for example, are based largely on normative self-reports and expert opinion. ${ }^{5051}$ Self-reported sleep data are prone to recall, reporting and social desirability bias and may provide estimates about $30-60 \mathrm{~min}$ more than actigraphy-measured sleep. $^{33} 52-54$ 


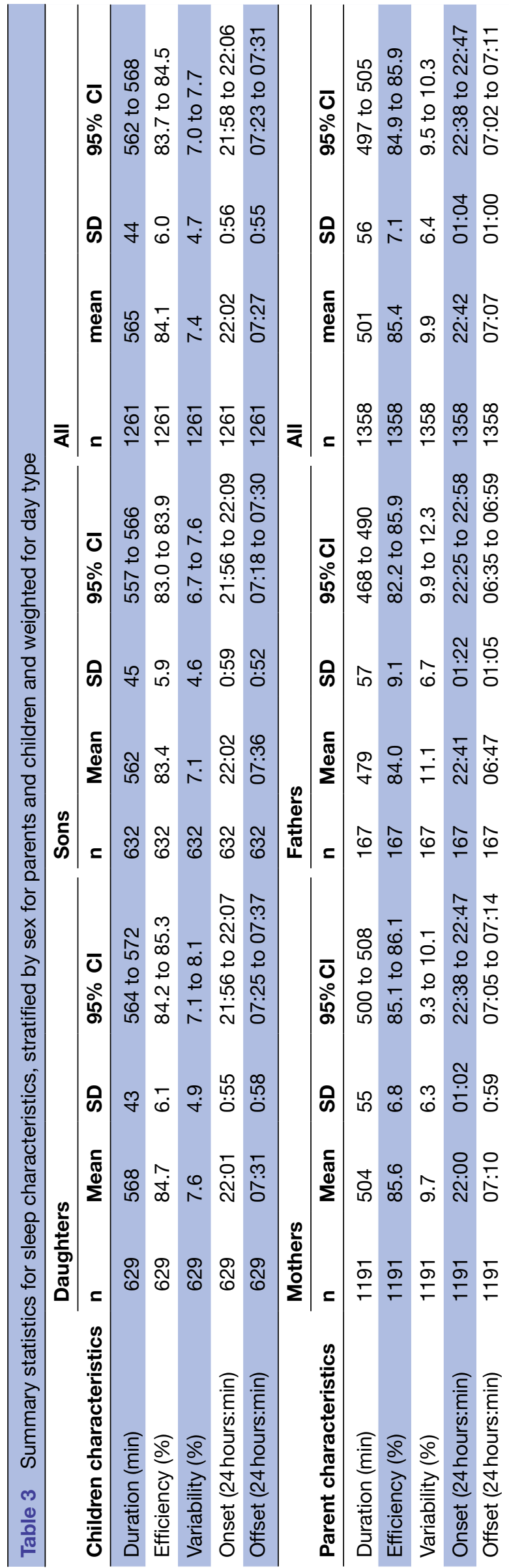

Meaning and implications for clinicians and policy makers Sleep characteristics

Given widespread concerns that society is chronically sleep deprived and that people are not sleeping enough, ${ }^{556}$ it may appear both promising and surprising that our study finds almost all Australian middle-aged parents and more than $70 \%$ of children aged 11-12 years meet widely accepted sleep recommendations. However, we also find that sleep quality, measured as sleep efficiency, was less than recommended in $42 \%$ of Australian middleaged parents and 55\% of children aged 11-12 years. Although there are currently no clear guidelines on sleep variability, several publications have suggested that consistent sleep schedules are important $\mathrm{t}^{57}$ and that day-to-day sleep variability should not exceed 1 hour. ${ }^{58} 59$ In this study, we find that $88 \%-92 \%$ of consecutive nights differ by less than 1 hour for parents and children, respectively.

Our findings are consistent with some, but not all, studies that objectively measure sleep duration and efficiency in children and adults of similar age groups. ${ }^{3460-65}$ In a metareview, Ohayon and colleagues ${ }^{34}$ reported sleep duration of approximately 450-550 $\mathrm{min}$ and 360-460 min (vs 565 and $501 \mathrm{~min}$ ) and sleep efficiency of approximately $87 \%-96 \%$ and $89 \%-98 \%$ (vs $85.4 \%$ and $84.1 \%$ ) of children and adults of similar age groups, respectively. Discrepancies may be partly related to different sample characteristics and the use of different measurement and accelerometer devices and analytical algorithms. Few studies have examined sleep variability. Findings consistent with our own have been reported by Spilsbury and colleagues, ${ }^{66}$ who examined self-reported sleep duration in children aged 10-11 years, while Moore and colleagues $^{67}$ reported higher sleep variability in objectively measured sleep, but in older children, which would be expected. Studies that have examined sleep variability of middle-aged adults have typically focused on clinical samples. ${ }^{20}$

Our study moves beyond the traditional approach of examining isolated sleep characteristics and provides normative sleep values for a wide range of sleep characteristics of a community-based sample, including sleep efficiency and variability, two sleep variables that are less frequently reported. This study is therefore in line with growing awareness that optimal ${ }^{51}$ healthy ${ }^{68}$ sleep may best be considered in terms of multiple sleep characteristic and is a step towards ongoing population sleep surveillance. ${ }^{69}$

\section{Parent-child concordance}

This study measures parent child concordance and found intergenerational concordance for all sleep characteristics: duration $(\mathrm{r}=0.22)$, onset $(\mathrm{r}=0.42)$, offset $(\mathrm{r}=0.58)$, day-to-day variability in duration $(\mathrm{r}=0.25)$ and efficiency $(\mathrm{r}=0.23)$. These findings are in contrast to previous studies, which reveal weaker correlations between self-reported parent and child sleep duration $(\mathrm{r}=0.02),{ }^{30}$ onset $(\mathrm{r}=0.08-0.39)^{30-32}$ and offset $(\mathrm{r}=0.02-0.38) .{ }^{30-32}$ Given that previous studies mostly rely on self-reported sleep 

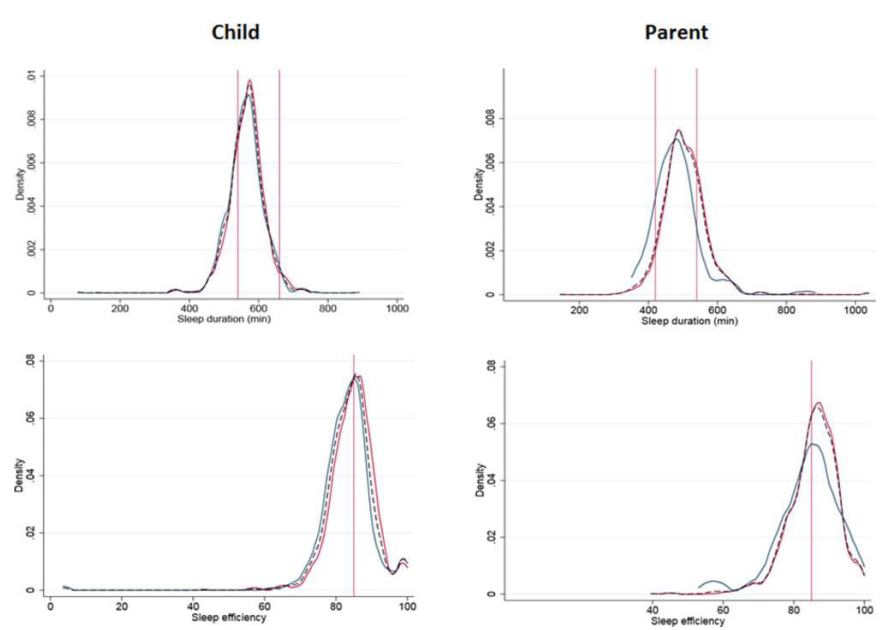

Figure 2 Density plots for sleep duration and sleep efficiency. Male sex (blue), female sex (red) and both sexes (thin dotted black line) plotted on the same graph for each outcome. $X$ and $Y$ scales common between child and parent, and between mean and maximum sleep variables. Vertical red lines indicate NSF sleep recommendations. The recommended range of appropriate sleep duration is 420-540 min for adults and 540-660 min for school-aged children. ${ }^{47}$ The NSF $^{35}$ recommends a sleep efficiency of $\geq 85 \%$ for both adults and children. NSF, National Sleep Foundation.

(open to recall, reporting and social desirability bias), our results should supersede earlier reports.

Twin studies have identified genetic factors as important determinants of habitual and disordered sleep, ${ }^{70}$ with the heritability of sleep duration and quality found to be $30 \%-40 \% .^{71}$ In our study, the strongest parent-child concordances were seen for those factors most likely to relate to household routines: bedtime, get-up time and day-to-day variability. Environmental factors may be extremely important determinants of sleep, given that sleep occurs within the constraints of the family home. For example, noise and lighting, as well as school and work start times, have been related to sleep and are likely to be strong determinants of family bed and rise times. ${ }^{69}$ Given that children aged 11-12 are dependent on their parents for care and transport, parents may, in a sense, be viewed as gatekeepers of their child's day. Efforts to improve children's sleep may therefore require a family-focused approach, whereby family sleep routines are considered, in addition to individual factors that may influence sleep (eg, homework).

\section{Unanswered questions and future research}

This study provides normative values for a wide range of sleep characteristics in a large, population-based sample of Australian middle-aged adults and children aged 11-12 years. These data provide reference values for future studies of LSAC participants and may contribute to a better understanding of the longitudinal association between sleep and health.

This study also identifies a need for further methodological studies to better understand and interpret objectively measured sleep characteristics. Calibration studies between different actigraphy device-algorithm combinations would be helpful to make meaningful comparisons of sleep measures attained from different methods. Given the growing awareness that sleep duration, as well as other characteristics of sleep, such as timing, variability and efficiency, may be important for health, future efforts are also needed to develop reference ranges for all objectively measured sleep characteristics.

Table 4 Parent-child concordance

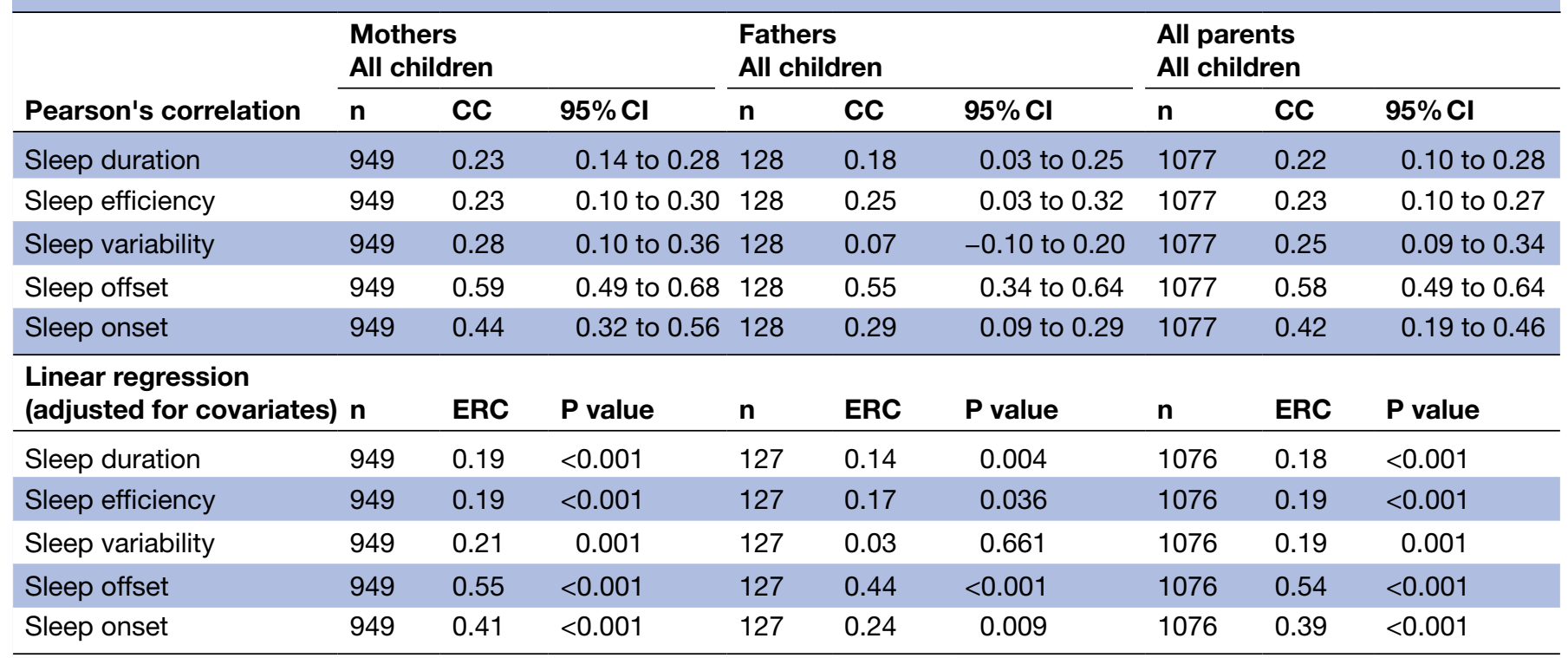

Covariates in adjusted linear regression models include parent and child ages, Disadvantage Index, type of day device worn, child sex and parent sex in the models for all parents.

$\mathrm{CC}$, correlation coefficient; ERC, estimated regression coefficient; $n$, sample size. 
To further decipher the role of environmental and genetic factors on population sleep, future studies that examine the concordance of sleep among biological and non-biological parent-child pairs, as well as differences in the concordance of sleep on weekdays and weekends, are needed. In the current study, we also report a weaker concordance for most sleep characteristics among fatherchild pairs compared with mother-child pairs; while a smaller father sample size may explain this finding, it is also possible that mothers continue to assume the traditional role of regulating household routines, such as meal times and children's bedtimes and rise times. However, further research is also needed in this area.

In conclusion, we provide normative data of a wide range of different sleep characteristics for Australian middle-aged parents and their children aged 11-12 years. Our study suggests that both environmental and genetic factors may be important determinants of sleep timing.

Acknowledgements This paper uses unit record data from Growing Up in Australia, the Longitudinal Study of Australian Children (LSAC). The study is conducted in partnership between the Department of Social Services (DSS), the Australian Institute of Family Studies (AIFS) and the Australian Bureau of Statistics (ABS). Research Electronic Data Capture (REDCap) electronic data capture tools were used in this study. More information about this software can be found at www. project-redcap.org. The authors thank the LSAC and CheckPoint study participants, staff and students for their contributions.

Contributors All authors conceptualised the manuscript. LM led the writing; FF, ACG, JM, MW and TO provided expert advice and critical review of the manuscript. ACG ran the analysis. MW is the principal investigator of the Child Health CheckPoint, planned the analyses and provided critical review of the manuscript. T0 is a study investigator involved in the conception and oversight of the Child Health CheckPoint.

Funding This work was supported by the National Health and Medical Research Council (NHMRC) of Australia (project grants 1041352 and 1109355), The Royal Children's Hospital Foundation (2014-241), the Murdoch Children's Research Institute (MCRI), The University of Melbourne, the National Heart Foundation of Australia (100660) and Financial Markets Foundation for Children (2014-055 and 2016-310). MW was supported by the NHMRC (1046518) and Cure Kids New Zealand. The MCRI administered the research grants for the study and provided infrastructural support (IT and biospecimen management) to its staff and the study, but played no role in the conduct or analysis of the trial. The Department of Social Services played a role in study design; however, no other funding bodies had a role in the study design and conduct; data collection, management, analysis and interpretation; preparation, review or approval of the manuscript; and decision to submit the manuscript for publication. Research at the MCRI is supported by the Victorian Government's Operational Infrastructure Support Program.

Disclaimer The findings and views reported in this paper are those of the authors and should not be attributed to DSS, AIFS or the ABS.

Competing interests None declared.

Patient consent for publication Not required.

Ethics approval The CheckPoint study protocol was approved by The Royal Children's Hospital Melbourne Human Research Ethics Committee (33225D) and the Australian Institute of Family Studies Ethic s Committee (14-26).

Provenance and peer review Not commissioned; externally peer reviewed.

Data sharing statement The Longitudinal Study of Australian Children datasets and technical documents are available to researchers at no cost via a licence agreement. Data access requests are co-ordinated by the National Centre for Longitudinal Data. More information is available at https://dataverse.ada.edu.au/ dataverse/lsac.

Open access This is an open access article distributed in accordance with the Creative Commons Attribution Non Commercial (CC BY-NC 4.0) license, which permits others to distribute, remix, adapt, build upon this work non-commercially, and license their derivative works on different terms, provided the original work is properly cited, appropriate credit is given, any changes made indicated, and the use is non-commercial. See: http://creativecommons.org/licenses/by-nc/4.0/.

\section{REFERENCES}

1. Cappuccio F, Miller M. Sleep, health, and society: from aetiology to public health. USA, 2010.

2. Park S, Cho MJ, Chang SM, et al. Relationships of sleep duration with sociodemographic and health-related factors, psychiatric disorders and sleep disturbances in a community sample of Korean adults. J Sleep Res 2010;19:567-77.

3. Xiang YT, Ma X, Lu JY, et al. Relationships of sleep duration with sleep disturbances, basic socio-demographic factors, and BMI in Chinese people. Sleep Med 2009;10:1085-9.

4. Olds TS, Maher CA, Matricciani L. Sleep duration or bedtime? Exploring the relationship between sleep habits and weight status and activity patterns. Sleep 2011;34:1299-307.

5. Astill RG, Van der Heijden KB, Van ljzendoorn MH, et al. Sleep, cognition, and behavioral problems in school-age children: a century of research meta-analyzed. Psychol Bull 2012;138:1109-38.

6. Lundahl A, Kidwell KM, Van Dyk TR, et al. A meta-analysis of the effect of experimental sleep restriction on youth's attention and hyperactivity. Dev Neuropsychol 2015;40:104-21.

7. Lovato N, Gradisar M. A meta-analysis and model of the relationship between sleep and depression in adolescents: recommendations for future research and clinical practice. Sleep Med Rev 2014;18:521-9.

8. Cappuccio FP, Taggart FM, Kandala NB, et al. Meta-analysis of short sleep duration and obesity in children and adults. Sleep 2008;31:619-26.

9. Cappuccio FP, D'Elia L, Strazzullo P, et al. Quantity and quality of sleep and incidence of type 2 diabetes: a systematic review and meta-analysis. Diabetes Care 2010;33:414-20.

10. Matthews KA, Pantesco EJ. Sleep characteristics and cardiovascular risk in children and adolescents: an enumerative review. Sleep Med 2016;18:36-49.

11. Cappuccio FP, Cooper D, D'Elia L, et al. Sleep duration predicts cardiovascular outcomes: a systematic review and meta-analysis of prospective studies. Eur Heart J 2011;32:1484-92.

12. Heffron T, Well S. Sleep Well, Be Well: A national health priority. 2014 http://sleepeducation.org/news/2014/05/16/sleep-well-be-well-anational-health-priority.

13. Perry GS, Patil SP, Presley-Cantrell LR. Raising awareness of sleep as a healthy behavior. Prev Chronic Dis 2013;10:10.

14. Chung KF, Cheung MM. Sleep-wake patterns and sleep disturbance among Hong Kong Chinese adolescents. Sleep 2008;31:185-94.

15. Holden C. Wake-up call for sleep research. Science 1993;259:305.

16. National Sleep Foundation. Lack of sleep is affecting Americans. 2014 https://sleepfoundation.org/media-center/press-release/lacksleep-affecting-americans-finds-the-national-sleep-foundation.

17. National Sleep Foundation. Sleep in America Poll: sleep in the modern family, 2014. 2014.

18. Adams R, Appleton S, Taylor A, et al. Australians have poor sleep habits. Report to the Sleep Health Foundation: 2016 Sleep Health Survey of Australian Adults: The University of Adelaide: The Adelaide Institute for Sleep Health, 2017.

19. Gradisar M, Gardner G, Dohnt H. Recent worldwide sleep patterns and problems during adolescence: a review and meta-analysis of age, region, and sleep. Sleep Med 2011;12:110-8.

20. Bei B, Wiley JF, Trinder J, et al. Beyond the mean: a systematic review on the correlates of daily intraindividual variability of sleep/ wake patterns. Sleep Med Rev 2016;28:108-24.

21. Rebelo-Pinto T, Pinto JC, Rebelo-Pinto $\mathrm{H}$, et al. Validation of a three-dimensional model about sleep: Habits, personal factors and environmental factors. Sleep Sci 2014;7:197-202.

22. Marinelli M, Pappa I, Bustamante M, et al. Heritability and genomewide association analyses of sleep duration in children: the EAGLE Consortium. Sleep 2016;39:1859-69.

23. Landolt $\mathrm{H}$. Genetic determination of sleep EEG profiles in healthy humans. In: Van Someren E, ed. Progress in Brain Research. The Netherlands, 2011.

24. Griefahn B, Marks A, Robens S. Noise emitted from road, rail and air traffic and their effects on sleep. J Sound Vib 2006;295:129-40.

25. Brand S, Hatzinger M, Beck J, et al. Perceived parenting styles, personality traits and sleep patterns in adolescents. J Adolesc 2009;32:1189-207.

26. Ohayon MM, Smolensky MH, Roth T. Consequences of shiftworking on sleep duration, sleepiness, and sleep attacks. Chronobiol Int 2010;27:575-89. 
27. Jiang $X$, Hardy LL, Baur LA, et al. Sleep duration, schedule and quality among urban Chinese children and adolescents: associations with routine after-school activities. PLoS One 2015;10:e0115326.

28. Hublin C, Kaprio J. Genetic epidemiology of sleep and sleep disorders. In: Paul S, Mehdi T, Michael T, eds. The genetic basis of sleep and sleep disorders: Cambridge University Press, 2013.

29. Butkovic A, Vukasovic T, Bratko D. Sleep duration and personality in Croatian twins. J Sleep Res 2014;23:153-8.

30. Gau SS, Merikangas KR. Similarities and differences in sleep-wake patterns among adults and their children. Sleep 2004;27:299-304.

31. Zhang J, Li AM, Fok TF, et al. Roles of parental sleep/wake patterns, socioeconomic status, and daytime activities in the sleep/wake patterns of children. J Pediatr 2010;156:606-12.

32. Iwata S, Iwata O, Matsuishi T. Sleep patterns of Japanese preschool children and their parents: implications for co-sleeping. Acta Paediatr 2013;102:e257-62.

33. Matricciani L. Subjective reports of children's sleep duration: does the question matter? A literature review. Sleep Med 2013;14:303-11.

34. Ohayon MM, Carskadon MA, Guilleminault C, et al. Meta-analysis of quantitative sleep parameters from childhood to old age in healthy individuals: developing normative sleep values across the human lifespan. Sleep 2004;27:1255-73.

35. Ohayon M, Wickwire EM, Hirshkowitz M, et al. National Sleep Foundation's sleep quality recommendations: first report. Sleep Health 2017;3:6-19.

36. Australian Institute of Family Studies. Growing Up in Australia: the Longitudinal Study of Australian Children. 2015 http://www. growingupinaustralia.gov.au/.

37. Edwards B. Growing up in Australia: the Longitudinal Study of Australian Children: entering adolescence and becoming a young adult. Family Matters 2014;95:5-14.

38. Sanson A, Johnstone R. The LSAC Research Consortium \& FaCS LSAC Project Team. Growing Up in Australia takes its first steps. Family Matters 2004;67:46-53.

39. Clifford SA, Davies S, Wake M. Child Health CheckPoint: cohort summary and methodology of a physical health and biospecimen module for the Longitudinal Study of Australian Children. BMJ Open 2019;9(suppl 3):3-22.

40. Wake M, Clifford S, York E. Introducing Growing Up in Australia's Child Health CheckPoint. Family Matters 2014;94:15-23.

41. Harris PA, Taylor R, Thielke R, et al. Research electronic data capture (REDCap)-A metadata-driven methodology and workflow process for providing translational research informatics support. J Biomed Inform 2009;42:377-81.

42. Migueles $\mathrm{JH}$, Cadenas-Sanchez $\mathrm{C}$, Ekelund U, et al. Accelerometer data collection and processing criteria to assess physical activity and other outcomes: a systematic review and practical considerations. Sports Med 2017;47:1821-45.

43. Fraysse F, Grobler AC, Muller J, et al. Physical activity and sedentary activity: population epidemiology and concordance in Australian children aged 11-12 years and their parents. BMJ Open 2019;9(suppl 3):136-46.

44. van Hees VT, Sabia S, Anderson KN, et al. A novel, open access method to assess sleep duration using a wrist-worn accelerometer. PLoS One 2015;10:e0142533.

45. Australian Bureau of Statistics. Census of population and housing: Socio-Economic Indexes for Areas (SEIFA). In: 2033.0.55.001 Cn, ed. 2011.

46. Ellul S, Hiscock R, Mensah F, et al. Longitudinal Study of Australian Children's Child Health CheckPoint Technical Paper 1: weighting and non-response. Melbourne: Murdoch Children's Research Institute, 2018.

47. National Sleep Foundation. How much sleep do we really need? 2015 https://sleepfoundation.org/how-sleep-works/how-muchsleep-do-we-really-need.

48. Hjorth MF, Chaput J-P, Damsgaard CT, et al. Measure of sleep and physical activity by a single accelerometer: can a waist-worn
Actigraph adequately measure sleep in children? Sleep Biol Rhythms 2012;10:328-35.

49. Zinkhan M, Berger K, Hense S, et al. Agreement of different methods for assessing sleep characteristics: a comparison of two actigraphs, wrist and hip placement, and self-report with polysomnography. Sleep Med 2014;15:1107-14.

50. Hirshkowitz M, Whiton K, Albert SM, et al. National Sleep Foundation's sleep time duration recommendations: methodology and results summary. Sleep Health 2015;1:40-3.

51. Matricciani L, Blunden S, Rigney G, et al. Children's sleep needs: is there sufficient evidence to recommend optimal sleep for children? Sleep 2013;36:527-34.

52. Gaina A, Sekine M, Chen X, et al. Weekly variation in sleep patterns: estimates of validity in Japanese schoolchildren. Sleep Biol Rhythms 2005;3:80-5.

53. Short MA, Gradisar M, Lack LC, et al. The discrepancy between actigraphic and sleep diary measures of sleep in adolescents. Sleep Med 2012;13:378-84.

54. Lauderdale DS, Knutson KL, Yan LL, et al. Self-reported and measured sleep duration: how similar are they? Epidemiology 2008:19:838-45.

55. Matricciani L, Olds T, Williams M. A review of evidence for the claim that children are sleeping less than in the past. Sleep 2011;34:651-9.

56. Matricciani LA, Olds TS, Blunden S, et al. Never enough sleep: a brief history of sleep recommendations for children. Pediatrics 2012;129:548-56.

57. National Sleep Foundation. Healthy sleep tips. $2017 \mathrm{https} / /$ sleepfoundation.org/sleep-tools-tips/healthy-sleep-tips.

58. He F, Bixler EO, Liao J, et al. Habitual sleep variability, mediated by nutrition intake, is associated with abdominal obesity in adolescents. Sleep Med 2015;16:1489-94.

59. Owens J, Mindell J. Sleep in children and adolescents, an issue of pediatric clinics - E-Book. 2011

60. Mcneil J, Tremblay MS, Leduc G, et al. Objectively-measured sleep and its association with adiposity and physical activity in a sample of Canadian children. J Sleep Res 2015;24:131-9.

61. Harrington SA. Relationships of objectively measured physical activity and sleep with BMI and academic outcomes in 8-year-old children. Appl Nurs Res 2013;26:63-70.

62. Spruyt K, Molfese DL, Gozal D. Sleep duration, sleep regularity, body weight, and metabolic homeostasis in school-aged children. Pediatrics 2011;127:e345-52.

63. Arora T, Broglia E, Pushpakumar D, et al. An investigation into the strength of the association and agreement levels between subjective and objective sleep duration in adolescents. PLoS One 2013;8:e72406

64. Kuula L, Pesonen AK, Martikainen S, et al. Poor sleep and neurocognitive function in early adolescence. Sleep Med 2015;16:1207-12.

65. Lauderdale DS, Knutson KL, Yan LL, et al. Objectively measured sleep characteristics among early-middle-aged adults: the CARDIA study. Am J Epidemiol 2006;164:5-16.

66. Spilsbury JC, Storfer-Isser A, Drotar D, et al. Sleep behavior in an urban US sample of school-aged children. Arch Pediatr Adolesc Med 2004;158:988-94.

67. Moore M, Kirchner HL, Drotar D, et al. Correlates of adolescent sleep time and variability in sleep time: the role of individual and health related characteristics. Sleep Med 2011;12:239-45.

68. Buysse DJ. Sleep health: can we define it? Does it matter? Sleep 2014;37:9-17.

69. Matricciani L, Bin YS, Lallukka T, et al. Past, present, and future: trends in sleep duration and implications for public health. Sleep Health 2017;3:317-23.

70. Barclay NL, Gehrman PR, Gregory AM, et al. The heritability of insomnia progression during childhood/adolescence: results from a longitudinal twin study. Sleep 2015;38:109-18.

71. Antic N, Rajaratnam S, Naughton M, et al. Sleep Medicine. 2017. 